\title{
EDITORIALE
}

\section{MARKET DRIVEN MANAGEMENT NELL'ECONOMIA DIGITALE}

\author{
Alberto Pastore*
}

\section{Prologo}

22 Febbraio 1988

Oggi sono particolarmente euforico. Per la prima volta la mia azienda, la Andersen Consulting, mi manda dal cliente da solo, per un incontro con il Direttore Amministrativo. Spero di cavarmela, dopotutto sono solo un Experienced Assistant Consultant e ho appena compiuto 26 anni! Dobbiamo a breve rilasciare il nuovo sistema di contabilità analitica, basato su un programma di una emergente software house tedesca, ma purtroppo ci sono dei problemi ancora da risolvere: le interfacce con i sottosistemi alimentanti non funzionano a dovere e il sistema di reporting, realizzato con una applicazione ad hoc, mostra inattese rigidità. Il Direttore del Centro Elaborazione Dati del cliente dice che il programmatore esperto in Cobol non è disponibile in questi giorni ma che intanto possiamo fare una analisi dei punti critici. Il viaggio per Vasto, sede della Società Italiana Vetro, è lungo ma ho appena ritirato la mia prima macchina, una Y10 nuova fiammante, per cui sarà piacevole attraversare l'Appennino con questa bella giornata di sole. E poi ... finalmente ho avuto in dotazione (solo per questa occasione) uno dei tre nuovissimi computer acquistati dalla sede. È portatile, una cosa del tutto innovativa. Ha la dimensione di una piccola valigia ma non pesa molto, circa 12 chili, ed è dotato di una capacità di elaborazione eccezionale: la ram è di 1 mega! Lo schermo è grande, saranno 10 pollici, ed il carattere insolito, arancione. Sarà molto utile per fare il verbale dell'incontro e per aggiornare la documentazione tecnica.

* Professore Ordinario di Economia e gestione delle imprese alla Sapienza Università di Roma. Email: alberto.pastore@uniroma1.it. 
12 Febbraio 2009

Due giornate davvero interessanti! È proprio valsa la pena di venire a Londra per la Digital Economy International Conference. Londra è sempre una delle città più affascinanti del mondo (insieme a Roma naturalmente) $e$ l'incontro è stato di altissimo livello: le esperienze che sono state raccontate dagli esponenti del mondo delle imprese davvero istruttive, la sintesi del Prof. Jerry Wind, intervenuto da Philadelphia in videoconferenza, è stata illuminante, la visione proposta da Chris Anderson affascinante, in qualche modo sconvolgente. Nel 2008 sono più di un miliardo e mezzo le persone connesse ad internet nel mondo, l'incidenza delle vendite online sul totale retail supera il $10 \%$ negli USA, le imprese che acquistano in rete nei principali paesi occidentali sono oramai la maggioranza. Di grande interesse, emblematici se non paradigmatici, i casi presentati da eBay, Amazon e Dell sulla co-creazione di valore da parte dei clienti-prosumer; altrettanto significativi, per altri versi, gli approcci fondati sull'e-business e sull'integrazione informativa della catena del valore di colossi e protagonisti dell'ambiente fisico come Wal-mart e Cisco; per non parlare delle straordinarie esperienze di pure players dot.com come Google e Yahoo! La velocità di diffusione dell'economia digitale è impressionante e nei prossimi anni interesserà, fino a pervaderli, ambiti geografici e settoriali che fino ad oggi sono rimasti parzialmente al margine. Cosa accadrà? Non è facile immaginarlo: la continua evoluzione delle sue forme rende complicato prevedere quali saranno gli effetti dello sviluppo della rivoluzione digitale sull'economia, sul modo di fare business, sulla vita delle persone, nella veste di consumatori e non. L'unica cosa certa è che siamo solo all'inizio e che i più grandi cambiamenti devono ancora avvenire, nel segno di alcune tendenze che cominciano a profilarsi all'orizzonte.

Aspettando l'ora del volo voglio completare l'articolo sul tema del convegno ed inviarlo subito via mail ad Annalisa per garantire i tempi di uscita della nostra rivista. Dovrei fare in tempo anche a collegarmi in video con l'aula del Master a Roma, per salutare Guido e ringraziarlo per essere venuto anche quest'anno a fare lezione da noi.

Speriamo che l'aereo sia in orario, alle 20,30 devo assolutamente essere a casa. Oggi è il compleanno di mia moglie e andiamo a teatro, dove recita Carlo in carne e ossa. Qui la separazione tra bit e atomi non è ancora arrivata! Accidenti, il regalo! Mi connetto immediatamente su Yoox dove troverò certamente qualcosa che le piacerà.

19 Febbraio 2029

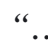

Una giornata stimolante ma faticosa oggi! Sono un po' stanco, penso che andrò a rilassarmi andando a trovare i miei nipoti. D'altro canto non sono più un giovincello, domani compio 67 anni! 
Fare oggi il diario del 19 febbraio 2029 non sarebbe corretto e comunque sarebbe molto arduo. Anche facendo ricorso alle previsioni dei guru dell'economia digitale e alla più fervida immaginazione risulterebbe assai complesso e aleatorio presumere degli scenari su un orizzonte temporale di così lungo termine.

D'altronde, per chi vive una rivoluzione è difficile interpretarne correttamente la portata e comprendere appieno la complessità del cambiamento. La rivoluzione tecnologica, infatti, può essere vista semplicemente come una serie di nuovi dispositivi, degli elementi frammentari, dispersi, privi di struttura; oppure, all'opposto, come una vera e propria palingenesi, il voltare pagina in modo netto, il passare dal vecchio al nuovo.

Alcune certezze le abbiamo: l'innovazione procede ad una velocità crescente, i mutamenti cui abbiamo assistito negli ultimi venti anni non saranno nulla a confronto con quelli che ci attendono nel prossimo futuro; la rivoluzione digitale è solo agli inizi, siamo in piena corsa, in uno scenario in rapido divenire, dove l'unica costante è il cambiamento. La direzione nella quale ci muoviamo è quella dell'economia post-industriale, della complessità, della collaborazione, dell'interdipendenza, della comunicazione; un terreno foriero di enormi potenzialità di sviluppo che ci pone delle sfide inedite, di grande portata.

\section{Business management nell'economia digitale}

L'economia digitale nasce e si sviluppa con la diffusione delle nuove Information and Communication Technologies (ICT), le quali offrono alle organizzazioni ed agli individui capacità di elaborazione e di comunicazione delle informazioni sempre maggiori a costi decrescenti. Si è avviato così un processo di sviluppo e di trasformazione dell'economia che da una parte sta cambiando radicalmente il modo con il quale le imprese fanno business e generano valore per i loro clienti, dall'altra sta modificando il ruolo del consumatore stesso nel sistema economico.

Mutuando l'approccio di Rullani ${ }^{1}$, possiamo descrivere lo sviluppo dell'economia digitale secondo tre prospettive distinte, tre fasi succedutesi temporalmente e logicamente, ma tra loro strettamente correlate e sintetizzate nel paradigma postfordista: new economy, net economy e knowledge economy.

La prima dimensione del fenomeno, comunemente qualificata come new economy, concerne la rapida, dirompente, crescita del settore ICT, dettata dalle eccezionali potenzialità offerte dalle nuove tecnologie, sempre

1. Rullani E., "New, net, knowledge economy: le molte facce del postfordismo", Economia e politica industriale, n. 110, 2001. 
più efficienti e meno costose, che diventano un driver fondamentale per incrementare la produttività dei sistemi economici e delle imprese. Il settore ICT si trasforma radicalmente in termini di logiche operative, di dimensioni, di apprezzamento (pur temporaneo) da parte dei sistemi finanziari; diventa "nuova economia", se osservato dal punto di vista dell'offerta.

Ma il vero cambiamento, l'effetto più dirompente, l'apertura alle più significative frontiere della rivoluzione digitale si ha con l'attivazione della net economy o economia dell'interazione. Essa emerge grazie alla capacità delle nuove tecnologie di offrire delle soluzioni ai problemi di business delle imprese, di produrre organizzazione, favorendo la connessione, l'interscambio informativo, l'interazione, la gestione delle relazioni, l'integrazione interaziendale dei processi gestionali. Tra gli effetti più rilevanti delle moderne ICT infatti si colloca la riduzione dei costi di transazione, fattore che dischiude un ampio ventaglio di soluzioni interorganizzative per la gestione di un business. L'economia dell'interazione sprigiona un enorme potenziale, dovuto da una parte alle esternalità di rete, per le quali il valore di una rete cresce in funzione del numero di partecipanti, e dall'altra alle economie di varietà, ovvero alle innumerevoli opportunità di combinazione delle risorse detenute da molteplici soggetti e orientate verso le più diverse finalità. Inoltre, le nuove tecnologie consentono di superare il tipico trade-off del potenziale comunicativo tra reach (ampiezza di soggetti coinvolti) e richness (ricchezza informativa, in termini di profondità, interazione e personalizzazione).

Nella net economy si osserva, dunque, il superamento della logica gerarchica e la diffusione di meta-organizzazioni economiche e sociali, forme organizzative a rete, filiere produttive composite basate su un'efficace ed efficiente divisione del lavoro, dettata dai principi della specializzazione e della partecipazione collaborativa. Il ruolo del consumatore in questo contesto muta radicalmente: egli diventa nodo dinamico della rete, capace di partecipare attivamente ai processi di esplorazione, creazione e fruizione del valore. Sfruttando queste potenzialità, si configurano dei sistemi di generazione del valore del tutto innovativi, lontani anni luce dai tradizionali paradigmi dell'economia d'impresa brick-and-mortar integrata.

Il terzo volto della rivoluzione digitale è quello della knowledge economy. Grazie alla capacità di gestione delle relazioni offerta dall'economia dell'interazione ed in funzione della destrutturazione della catena del valore, i soggetti economici tendono a specializzare il proprio ruolo, definendo la loro attività core su porzioni più ridotte della catena medesima ed incrementando al tempo stesso la profondità delle loro competenze distintive in quello specifico ambito. Gli investimenti in conoscenza (ricerca e sviluppo, innovazione, formazione, ecc.) diventano ancora più strategici, i rischi si riducono, mentre gli ambiti di applicazione della stessa si moltiplicano, in funzione dell'apertura di mercati globali e delle opportunità di riuso intensivo, consentendo di conseguire quelle economie di scala che nell'economia digitale hanno una ri- 
levanza fondamentale. A livello meta-organizzativo, si sviluppano forme di intelligenza collettiva, di condivisione della conoscenza nell'ambito delle reti, che sovente costituiscono la fonte primaria del vantaggio competitivo.

L'economia digitale, dunque, si configura come l'ambiente elettivo per lo sviluppo del paradigma post-fordista, un contesto dove l'informazione, l'interazione, la conoscenza, la collaborazione costituiscono la linfa vitale del divenire dei sistemi economici, sia a livello macro che a livello micro. L'impresa vincente dell'era digitale è aperta, proiettata a livello globale, ha un rapporto dinamico e co-creativo con tutti i suoi stakeholder, soprattutto con i clienti, condivide le risorse, fa leva sulla collaborazione di massa.

\section{Marketing e whole relationship management nell'economia digitale}

Alla luce di quanto sopra argomentato, appare chiaro come il marketing stia subendo un'evoluzione paradigmatica in funzione delle opportunità offerte dall'economia digitale.

Osservando, anzitutto, gli effetti del fenomeno della digitalizzazione dell'economia sui principali protagonisti del processo di marketing, evidenziamo come i principali mutamenti riguardino evidentemente sia la sfera degli acquirenti/consumatori che quella delle imprese.

Nell'era digitale gli acquirenti/consumatori dispongono di un enorme potenziale informativo, possono interagire con i fornitori e con altri consumatori, sono più maturi ed esigenti, hanno maggiore consapevolezza delle alternative di offerta e dei relativi prezzi, possono scegliere tra un'infinita varietà di prodotti e servizi. In buona sostanza, il potere di mercato tende a spostarsi dai produttori e dai distributori verso gli acquirenti/consumatori, $\mathrm{i}$ quali sono in grado di partecipare attivamente e consapevolmente alla definizione delle caratteristiche della loro personale market solution, in termini di prodotti e servizi, prezzi, modalità distributive, forme di comunicazione.

Nella prospettiva dell'impresa le principali innovazioni nell'approccio al mercato riguardano la possibilità di migliorare le loro capacità di sensing, stimolare la partecipazione attiva dei consumatori, ridurre il time to market, raggiungere ampi target con un elevato grado di personalizzazione, realizzare compiutamente una strategia relazionale.

Gli effetti della digitalizzazione dell'economia sul marketing, però, devono essere letti ed interpretati anche a livello di sistema. Le ICT, infatti, sono uno dei vettori fondamentali che favoriscono l'emersione di un approccio al mercato di tipo olistico $^{2}$, fondato sulla connessione ed interazio-

2. Kotler P., Jain D. C., Maesincee S., "Marketing Moves. A new approach to profits, growth and renewal", Harvard Business School Press, 2002. 
ne tra l'impresa, le sue componenti interne, i suoi clienti, i suoi business partner (fornitori, distributori, partner orizzontali), per una gestione efficace, efficiente ed integrata del processo di analisi, creazione e diffusione del valore.

La separazione tra gli aspetti fisici e quelli informativi, tra atomi e bit, determina la destrutturazione della catena del valore: nel mondo digitale, più che in quello materiale, il valore è creato non solo dall'impresa ma anche dagli altri soggetti che fanno parte della rete, in primis dagli acquirenti/consumatori. Ciascuna attività può essere fatta da specialisti, in grado di svolgerla meglio, a costi inferiori, in minore tempo, con maggiore efficacia. L'impresa focale della rete deve dunque disegnare la catena esterna del valore definendo le risorse necessarie, individuando i soggetti partecipanti, stabilendo i ruoli e le relazioni. Dopodiché essa deve porre in atto la sua strategia di whole relationship management.

La prima dimensione è quella del customer relationship management: attivare e gestire una relazione di lungo termine con i clienti, basata sull'ascolto, la fiducia, l'adattamento reciproco; favorire la partecipazione attiva dei consumatori, trasformarli in prosumers per la co-definizione dell'offerta e la produzione di contenuti.

La seconda prospettiva riguarda l'internal resource management: l'impresa si muove dall'interno verso l'esterno, ovvero promuove le proprie dinamiche competitive attraverso le strutture, i processi organizzativi, le persone; è fondamentale pertanto attuare efficaci strategie di knowledge management e di marketing interno, al fine di disporre di competenze core qualificanti e di collaboratori opportunamente formati e motivati.

Il terzo ambito si riferisce al network management: nel disegno della piattaforma del network occorre selezionare i business partner in funzione delle loro capacità distintive, del contributo che possono dare alla generazione del valore, delle affinità culturali, valoriali e strategiche; nella gestione della relazione occorre perseguire la massima cointeressenza per garantirsi una co-evoluzione sinergica degli attori.

Il quarto livello concerne il social and istitutional relationship management: l'impresa si rivolge alle istituzioni ed alla società civile, consapevole di interpretare un ruolo di cittadinanza collettiva, fondando il suo approccio sui principi della corporate social responsibility; in questo quadro reputazione, immagine istituzionale, posizionamento sul mercato si fondono, contribuendo alle sorti competitive dell'impresa.

Infine, vogliamo citare un nuovo ambito nel quale le imprese si trovano sempre di più a dovere interagire con il pubblico dei consumatori, quello dei cosiddetti consumer generated media (CGM), dove, grazie alle nuove forme delle ICT i partecipanti interagiscono tra di loro producendo direttamente contenuti comunicazionali. Tali contesti sono caratterizzati da una comunicazione "orizzontale", capace di condizionare l'immagine di una 
marca, influenzare i comportamenti d'acquisto, attivare processi virali di grande risonanza, in una parola incidere sulle performance di mercato dell'impresa. Essa deve pianificare dunque un customer to customer relationship management, ascoltando le "discussioni" che si svolgono nei CGM, partecipandovi direttamente, fino a promuovere la costituzione di nuovi contesti e alimentandoli attraverso il diretto coinvolgimento del management e dei dipendenti.

Nell'economia digitale, dunque, tende ad affermarsi il paradigma del marketing olistico o del market driven management, secondo il quale la cultura del marketing va a permeare l'intera organizzazione, andando a ricoprire un ruolo fondamentale nel governo dell'impresa e nello sviluppo della sua strategia relazionale. Packard diceva che "il marketing è troppo importante per lasciarlo alla funzione marketing". Nell'era dei bit potremmo andare oltre, affermando che il marketing è troppo importante per restare all'interno dei confini dell'impresa e non fondarsi sul contributo di tutti gli stakeholders, cogliendo tutte le opportunità offerte dalla rivoluzione digitale. 